\title{
Effectiveness of Rehabilitation Nursing versus Usual Therapist-Led Treatment in Patients with Acute Ischemic Stroke: A Randomized Non-Inferiority Trial
}

\author{
Jianmiao Wang (D) \\ Yuping Zhang' \\ Yuanyuan Chen ${ }^{2}$ \\ Mei Li (D) \\ Hongyan Yang ${ }^{2}$ \\ Jinhua Chen ${ }^{2}$ \\ Qiaomin Tang ${ }^{2}$ \\ Jingfen Jin ${ }^{1,3}$
}

'Nursing Department, The Second Affiliated Hospital of Zhejiang University School of Medicine, Hangzhou, Zhejiang Province, People's Republic of China; ${ }^{2}$ Neurology Department, The Second Affiliated Hospital of Zhejiang University School of Medicine, Hangzhou, Zhejiang Province, People's Republic of China; ${ }^{3}$ Changxing Branch Hospital, The Second Affiliated Hospital of Zhejiang University School of Medicine, Huzhou, Zhejiang Province, People's Republic of China
Correspondence: Jingen Jin

The Second Affiliated Hospital of Zhejiang University School of Medicine, No. 88 Jiefang Road, Shangcheng District, Hangzhou, Zhejiang Province, 310009,

People's Republic of China

Fax +86-057I-87783778

Email zrjzkhl@zju.edu.cn
Purpose: To determine the effectiveness of rehabilitation nursing program interventions in patients with acute ischemic stroke.

Patients and Methods: An assessment-blinded randomized controlled trial was conducted at a tertiary referral hospital in China. Eligible patients were stratified according to their weighted corticospinal tract lesion load and then randomly assigned to an experimental group $(n=121)$ or a control group $(n=103)$. The experimental group received rehabilitation nursing from well-trained, qualified nurses (30 minutes per session, two sessions per day for seven consecutive days). The control group received therapist-led rehabilitation with the same timing and frequency. Comparative analysis of the primary outcomes was performed to determine non-inferiority with a predetermined non-inferiority margin. The primary outcomes were the Motor Assessment Scale, Fugl-Meyer Assessment, and the Action Research Arm Test assessed at baseline and after seven days of treatment. The secondary outcomes were the modified Barthel Index, the National Institutes of Health Stroke Scale, and the modified Rankin Scale, evaluated before and after the intervention and at 4 and 12 weeks of follow-up.

Results: Two hundred participants completed the trial. In both groups, all outcomes improved significantly after seven days and at follow-ups. The rehabilitation nursing program was non-inferior to therapist-led treatment with lower $95 \%$ confidence limits beyond the margins for primary outcomes $(P<0.001)$.

Conclusion: Both treatments had comparable effects; however, no definite conclusion could be drawn. Adequately powered studies are required.

Keywords: rehabilitation, nursing, acute ischemic stroke, motor function

\section{Introduction}

Stroke is the leading cause of mortality and disability worldwide; $87 \%$ of all deaths from stroke and $89 \%$ of all stroke-related disability-adjusted life-years occur in low- and middle-income countries (LMICs). ${ }^{1}$ In contrast to the global downward trend, the incidence is rising in LMICs, with approximately 2.4 million new stroke cases each year in China; more than one-third of acute ischemic stroke (AIS) patients die or become disabled within three months or one year. ${ }^{2}$ The high risk of disability and dysfunction may be related to the aging of the population. ${ }^{3}$ By 2050 , one in six people in the world will be 65 years of age or older. ${ }^{4}$ COVID-19related ischemic stroke leads to worse functional outcomes and higher mortality. ${ }^{5}$ Secondary stroke risk increased not only because of the disease characteristics of 
COVID-19 but also because of lack of physical activity due to isolation or restriction of access to treatments. ${ }^{6}$ Due to limited medical resources and isolation policies, access to services has been significantly reduced, and the burden of disability has increased further.

The functional limitations of the trunk and limbs associated with stroke reduce the ability to participate in activities of daily living (ADLs), requiring assistance with eating, drinking, moving, toileting, performing personal hygiene tasks, dressing, and grooming, ${ }^{7}$ which seriously affects the quality of life of stroke patients. ${ }^{8}$ The severity and variety of disorders in patients after stroke relate to the site and infarct size, and motor dysfunction is the principal problem. The leading causes are damage to the corticospinal tract (CST) and brain motor centers. ${ }^{9}$ The CST is the most critical motor control pathway that affects motor function recovery and outcome from a stroke. Motor function training in the acute stage (within the first two weeks ${ }^{10}$ ) can increase the structural integrity of the ipsilesional CST. ${ }^{11}$

After a stroke, rehabilitation is essential to help survivors achieve an optimal functional level and prevent or delay future functional decline. ${ }^{12}$ In the first days and weeks, the brain responds most quickly to the stimulus of motor training, ${ }^{13}$ In appropriate amounts, early training aids recovery and improves outcomes and quality of life. Early rehabilitation interventions in acute care settings are critical to optimizing the recovery potential in repair windows and prevent various complications secondary to the disability. Guidelines recommend providing early rehabilitation services for hospitalized stroke patients in an organized, multi-professional stroke care environment. ${ }^{10,14}$

Nevertheless, there are few practice guidelines or clinical pathways, and published guidelines do not guarantee effective implementation in practice. ${ }^{15}$ There are gaps between the best evidence and current practice that are not conducive to clinical intervention implementation or patient benefits. The consensus reached in the second stroke recovery and rehabilitation roundtable was to determine knowledge translation priorities and take specific actions to deal with the practice gaps. ${ }^{16}$

Inadequate resources for rehabilitation services are the main reason why clinical practices do not follow guidelines well. Many LMICs provide some rehabilitation care in acute settings, and transitional and community rehabilitation are rare. ${ }^{16}$ The proportion of stroke patients in LMICs receiving rehabilitation treatment is too small, and rehabilitation or treatment within seven days after stroke is also limited. ${ }^{17}$ Currently, there are about 10,000 rehabilitation physicians and 20,000 rehabilitation therapists in China, with an average of 1 to 2 per 100,000 people, much lower than the 40 to 70 per 100,000 in developed countries. ${ }^{18}$ According to the World Health Organization's Rehabilitation 2030 report, the numbers of rehabilitation practitioners, are far below those of highincome countries, while data on rehabilitation nurses are not available. ${ }^{19}$ Access to related rehabilitation services and staffing are systemic issues that need to be prioritized, and it is recommended that solutions be implemented to address these issues in the context of local realities to improve the quality of life of stroke patients. ${ }^{16}$

In LMICs, localized measures to improve functional outcomes after stroke with low-cost, resource-saving physical rehabilitation interventions are possible. ${ }^{20}$ There is evidence that aerobic programs and rehabilitation assistants increase the intensity of rehabilitation. ${ }^{16}$ As part of a multidisciplinary team, nurses play critical roles in facilitating stroke recovery, and recognizing their valuable contributions is essential. ${ }^{21}$ Nurses provide rehabilitation services in nursing homes and community rehabilitation centers and should also provide rehabilitation services in the acute phase. ${ }^{22}$ Primary care nurses' complex interventions increased the number of objectively measured stepcounts and moderate-to-intense physical activity. ${ }^{23}$ Enrolling nurses in task-oriented training can create more opportunities for patients to practice meaningful functional tasks outside of their regular treatment sessions. When nurses incorporate rehabilitation goals into nurses' daily care, they also improve patient independence. ${ }^{24}$ However, in current clinical practice, nurses pay more attention to maintaining safe care and preventing potential problems, including falls; there is limited practical nursing evidence in the vital areas, including mobility. ${ }^{25}$

There is no consensus on acute rehabilitation nursing guidelines or practice activities. ${ }^{26}$ Stroke nursing includes good limb placement, turning over, and out-of-bed mobilization education. Rehabilitation principles should be more integrated into practice. Because the establishment of acute rehabilitation nursing is best customized locally to match available resources. ${ }^{27}$ We developed a rehabilitation nursing program to improve motor function. Due to insufficient evidence for interventions, based on expert opinions and combined with feasibility study results, we considered factors that facilitate or hinder implementation. The main components of rehabilitation nursing interventions include physical therapy (PT), occupational therapy (OT), ADLs, 
following the principles of repetitive task-oriented training and patient-centered individualization implementation, as described in our protocol. ${ }^{28}$ Our research was motivated by the question of whether rehabilitation nursing interventions are effective, and if so, how effective are they compared to rehabilitation provided by therapists in current practice?

While standard treatments already exist, some therapies may be safer, more convenient, or less expensive with similar efficacy. An educational training program for nurses improved their knowledge and practice in clinical settings and improved ADLs and self-care abilities for stroke patients. $^{29}$ The results of the cost-effectiveness analysis provide evidence that nursing interventions can save costs for ischemic patients. ${ }^{30}$ Patients hospitalized in the acute phase are more likely to have access to nurses; therefore, it is possible to conduct a comparative study of rehabilitation nursing interventions with therapies used in practice.

This trial aimed to identify an option with comparable efficacy rather than superior efficacy. Non-inferiority trials attempt to determine whether a new treatment is inferior to a reference treatment and define a predetermined noninferiority margin $(\delta) .{ }^{31}$ For this reason, it makes sense to use a non-inferiority trial design when comparing the effects of nurse-led rehabilitation and therapist-led rehabilitation. Because multidisciplinary team early rehabilitation is the guideline-recommended treatment, it would be unethical to use a placebo or no-treatment control in the study. $^{32}$ This study could not be designed as a three-arm trial that included a blank control.

Therefore, our objective was a non-inferiority comparison between a rehabilitation nursing intervention and a therapist-led treatment regarding motor function assessments (the primary outcomes). Sequence tests and secondary outcomes were assessed for superiority.

\section{Patients and Methods Trial Design}

This single-blind, randomized, prospective, non-inferiority trial was conducted in three wards of neurology departments at a tertiary hospital in China. We compared a rehabilitation nursing program intervention with usual therapist-led treatment. The Human Research Ethics Committee of the Second Affiliated Hospital of Zhejiang University School of Medicine approved the trial protocol (No. 2018-112), and all participants were informed about the purpose of the trial and provided informed written consent. The trial protocol was registered prospectively in the Clinical Trials.gov (NCT03702452) and was modified after peer review. Detailed information was published previously. $^{28}$

\section{Participants}

Patients were recruited within $24 \mathrm{~h}$ of admission and grouped according to inclusion criteria. Rehabilitation began the day after enrollment for mild to moderate stroke, $48 \mathrm{~h}$ after vital signs were stable and neurological damage was no longer progressive for severe stroke. Detailed inclusion and exclusion criteria were published previously. $^{28}$ Briefly, we included patients with AIS at risk for motor impairment and admitted to the hospital within seven days of the initial stroke symptom onset. Critical inclusion criteria included age (18-90 years old) and consciousness level 0 or 1 on the National Institutes of Health Stroke Scale (NIHSS) score. Exclusion criteria included a successful response to intravenous thrombolysis, hemorrhagic transformation, restricted mobilization, disability, or other conditions that affected the recovery of limb function, and other circumstances that prevented patients from cooperating with the researchers. This study complied with the guidelines of the Declaration of Helsinki.

\section{Intervention}

Patients received rehabilitation nursing training items based on the modified Barthel Index (MBI) score, and the score was classified into five grades in 20-point increments (entirely dependent, heavily dependent, moderately dependent, mildly dependent, and basic self-care). The training items were selected from the contents of the corresponding levels, increasing complexity and the number of training items as dependency decreased. The interventions included PT like Bobath therapy, bridge exercise, passive and active limb movement training, articular mobility training, sit-up training, transfer training, balance training, sit-to-stand training, walking training, stepping training, limb weight training, OT including repetitive task-oriented training (wiping tables, transferring screw caps, and picking up beans), ADL training for dressing and undressing, drinking, and others (for details, please see our article in Chinese ${ }^{33}$ or the protocol ${ }^{28}$ ). Each training item was taught or implemented by a nurse in addition to usual rehabilitation nursing. A session included 30 minutes of training, two sessions per day for seven consecutive days. This was the appropriate duration of 
intervention as the average hospital length of stay (LOS) of AIS in China is about 14.47 days. ${ }^{34}$ It was sufficient to detect a significant MBI improvement. ${ }^{35}$ The rehabilitation program's content was adjusted according to the grading level of MBI on the third day after the intervention.

Each training session was carried out when patients were available and adhered to the principle that patients would not experience tired during the process. We maintained a provision to prescribe individualized (referenced MBI grading level and patient preference and the body impairment) rehabilitation nursing interventions to maximize ADL principles.

\section{Control}

Before the trial, the clinical pathway for neurology ward rehabilitation included first assessing functional impairment, the severity of stroke and ADLs, and developing rehabilitation treatment plans and goals. After the patient is stabilized, the doctor prescribes usual therapist-led rehabilitation treatment and usual rehabilitation nursing (good limb placement, turning over, and out-of-bed mobilization education), with no requirement for frequency or intensity of treatment. The control group received the usual rehabilitation by a therapist, with the start time, duration, and frequency of treatment consistent with the intervention group. The content of usual rehabilitation training was the same as those delivered in the experimental group, but was less specific. The treatments involved more PT, including muscle strength training, limb resistance training, anti-spasm training, knee extension, and ankle plantar flexion training to induce separation movement, gait training, trunk control, and postural correction training. There was also OT such as grasping training, coordination and active movement training of the affected limbs and hands, and ADL training (for details, see the protocol ${ }^{28}$ ).

\section{Outcomes}

Primary outcomes were three motor function assessment tools that have excellent reliability and construct validity: the Motor Assessment Scale (MAS) to measure progress; ${ }^{36}$ Fugl-Meyer Assessment (FMA); ${ }^{37}$ and the Action Research Arm Test (ARAT) ${ }^{38}$ assessed at baseline and after seven days of treatment. The secondary outcomes were MBI, NIHSS, and the modified Rankin Scale (mRS) assessed at baseline (t0) and after seven days of treatment (t1), and at $4(\mathrm{t} 2)$, and 12 (t3) weeks of follow-up. These scales comprised domain and item numeric rating scores. Scoring was administered in- person by trained research nurses who were blinded to group assignment.

We also recorded socio-demographic and clinical data obtained from electronic medical record systems. We asked patients to report any unintended harms and severe adverse events directly during hospitalization or followup. Severe adverse events such as recurrence of stroke or death were recorded in detail by the nurse and reported to the data monitoring committee.

\section{Sample Size}

There is no widely accepted minimal clinically important difference (MCID) for the motor function assessment scale. A predefined margin of non-inferiority $(\delta)$ of 6.94 assumed $50 \%$ of the standard deviation (SD) in the control group from our preliminary study (owing to the unknown efficacy of positive control over placebo in the superiority test). We estimated that a sample size of 134 (67 patients per group) would provide $90 \%$ power (one-tailed $\alpha$ of 0.025) to detect a difference in MAS scores in patients with weighted corticospinal tract lesion load (wCST-LL) $<$ $2 \mathrm{~mL}$ between groups. The sample size in the wCST-LL $\geq$ $2 \mathrm{~mL}$ group is approximately half of wCST-LL $<2 \mathrm{~mL}$ group (67 patients), considering approximately 10\% attrition, a minimal sample size of 224 subjects was required. This is described in detail in the protocol. ${ }^{28}$

\section{Randomization}

We allocated patients into large or small groups using wCST-LL. Patients in each group were randomly assigned to the experimental (rehabilitation nursing intervention) or control (therapist-led treatment) group using a random numbers generator.

\section{Blinding and Concealment of Allocation}

The researchers assessing outcomes were blinded to group allocation. The randomization table was available to one researcher (the coordinator) who conducted the allocation, evaluation, and intervention process independently using a remote web-based system like a centralized randomization system. Doing so avoided selection bias and ensured allocation concealment. The head nurses coordinated the time for treatment and evaluation to avoid assessors discovering the allocation. The allocation table was made invisible to maintain blindness until the end of the trial and data analysis. The statistical analysis plan was not changed after the data were unblinded. 


\section{Statistical Methods}

Descriptive statistics were applied, reported as numbers and percentages for categorical variables and as means with SD for continuous variables. Between-group data were compared using the independent $t$-test, the Chi-square, or the Fisher exact test. The non-parametric data were presented as median (interquartile range, IQR) using the Mann-Whitney $U$-test or Friedman test as appropriate. The statistical analysis followed both the modified intention-to-treat (mITT) and the per-protocol (PP) approach. ${ }^{39}$ Missing values were imputed using the last observation carried forward method.

Reporting followed the CONSORT extension statement for non-inferiority trials. ${ }^{31}$ Non-inferiority at $\alpha$ is 0.025 in the one-tailed $t$-test, and the lower limit of the $95 \%$ confidence interval (CI) of the difference was within the presupposed non-inferiority margin only performed for the primary outcome measures. If the non-inferiority evidence rejects the null hypothesis, a superiority test would be conducted following the principle of sequential testing. The repeated measurement data of secondary outcome measures were analyzed using Generalized Estimating Equations (GEE), adjusted for age, sex, and the wCST-LL group. The overall alpha was 0.025 for both non-inferiority and superiority testing. The power calculation was performed using a noninferiority test of the difference of two means in PASS 11. Data analysis was performed using SPSS V.26.0 (IBM Corporation, US) and NCSS V.12.0.

No interim analyses were planned or performed. Subgroups of wCST-LL, age, and other indicators were compared in a post hoc analysis (beyond the scope of the present study).

\section{Results}

\section{Participant Flow}

The study flow of participants is depicted in Figure 1. A total of 1446 stroke patients were screened for participation. Of the 224 patients enrolled in this study, 121 were randomly assigned to the rehabilitation nursing group and 103 to the control group; 209 participants completed the treatment (108 completed the intervention, and 101 were in the control group). During follow-ups, nine dropped out (three in the experimental group and six in the control group); 200 attended all outcome assessments. Reasons for dropout are listed in detail. The main reason for dropping out was that the patient could not be reached by telephone. Two patients discontinued because they did not wish to continue the study or died (Figure 1).

\section{Recruitment}

From December 2018 to June 2020, this study achieved the required sample size without early termination.

\section{Baseline Data}

Demographic and clinical data are displayed in Table 1. The baseline variable distributions were similar, and outcome measures between the two groups showed no significant differences (all $P>0.05$ ). No significant differences were found between small- and large- wCST-LL stratified groups. These results suggest that the groups were comparable at baseline (Table 1).

\section{Numbers Analyzed}

After seven days of treatment, outcome assessment was completed for 108 and 101 patients in the experimental and control groups, respectively; the remaining nine participants dropped out. All randomized patients with outcome data $(n=209)$ and patients complying with the protocol and attending final secondary outcome assessment $(n=200)$ were entered separately into final mITT or PP analyses.

\section{Primary Outcomes and Estimation}

The non-inferiority of the experimental group to the control group over seven days of treatment could be claimed because the lower limit of the $95 \%$ CIs was greater than -6.94 in MAS, -12 in FMA, and -10 in the ARAT (Figure 2A and 2B). The null hypothesis was rejected by the Wilcoxon Rank-Sum Location difference test for non-inferiority $(P<0.001)$. Although the lower limit of the $95 \%$ CIs fell outside of zero, the subsequent superiority test could not be demonstrated because the median difference between groups after seven days were not statistically significant in PP analyses (MAS: $P=$ 0.032, FMA: $P=0.047$, ARAT: $P=0.056$, all $P>$ 0.0167 ) (Table 2) and in mITT analyses (MAS: $P=$ 0.032, FMA: $P=0.030$, ARAT: $P=0.047$, all $P>$ 0.0167) (Supplementary Table S1).

Based on the change margins calculated with the same method and data of MAS, FMA, and ARAT, the noninferiority hypothesis of the three primary outcomes changes was also established $(P<0.001)$. There were significant between-group differences in the MAS, FMA, and ARAT changes in PP analyses (change in MAS: $P=$ 0.003, change in FMA: $P=0.004$, change in ARAT: $P=$ 


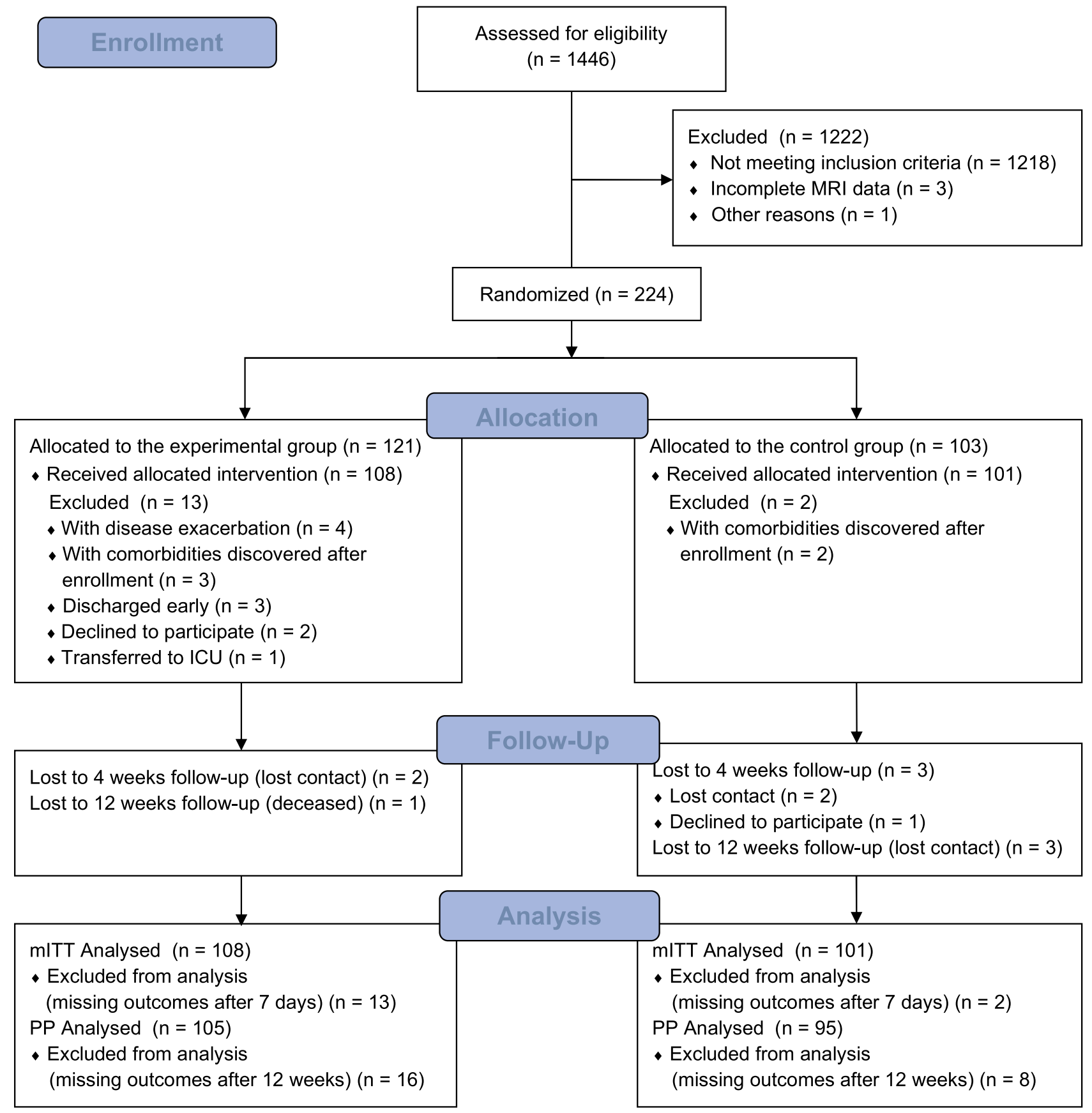

Figure I Flow chart of participants.

Abbreviations: mITT, modified intention-to-treat, all participants followed the random allocation with outcome data; PP, per-protocol, participants followed the random allocation with outcome data and attending the final outcome assessment.

0.001 , all $P<0.0167$ ) and mITT analyses (Table 2 and Supplementary Table S1).

\section{Secondary Outcomes and Estimation}

In PP analysis, the four-time-point GEE analysis showed significant improvement in MBI, NIHSS, and mRS scores over time (all $P<0.001$ ); however, there were no significant group differences for any of the outcomes (all $P>0.05$ ). There was a significant difference in the MBI interaction effect between time and group $(P=0.001)$ and no significant difference for NIHSS or mRS (Table 3). In mITT analysis for GEE, the results were broadly consistent, but showed a significant interaction effect in MBI, NIHSS, and mRS (all $P<0.0167$ ) (Supplementary Table S2). 
Table I Baseline Demographic and Clinical Characteristics Between Groups

\begin{tabular}{|c|c|c|c|}
\hline Variables & $\begin{array}{l}\text { Experimental Group } \\
(n=108)\end{array}$ & $\begin{array}{l}\text { Control Group } \\
(n=101)\end{array}$ & $P$ value \\
\hline Age (yrs), mean $\pm S D$ & $64.35 \pm 10.59$ & $61.36 \pm 15.27$ & 0.287 \\
\hline Male, n (\%) & 79 (73.1\%) & $68(67.3 \%)$ & 0.686 \\
\hline Stratification indicator, n (\%) & & & 0.459 \\
\hline wCST-LL $<2 \mathrm{~mL}$ & $59(54.6 \%)$ & $50(49.5 \%)$ & \\
\hline$w C S T-L L \geq 2 m L$ & $49(45.4 \%)$ & $5 \mathrm{I}(50.5 \%)$ & \\
\hline Time from onset to enrollment (d), mean $\pm S D$ & $3.81 \pm 1.61$ & $4.07 \pm 1.81$ & 0.492 \\
\hline \multicolumn{4}{|l|}{ Muscle strength on hemiplegic side, median (IQR) } \\
\hline Upper limb & $2.00(1.00-4.00)$ & $2.00(0.50-4.00)$ & $0.772^{\ddagger}$ \\
\hline Lower limb & $3.00(2.00-4.00)$ & $3.00(2.00-4.00)$ & $0.510^{\ddagger}$ \\
\hline Education, $\mathrm{n}(\%)$ & & & $0.07 I^{\ddagger}$ \\
\hline Illiteracy & $10(9.3 \%)$ & $\mathrm{II}(10.9 \%)$ & \\
\hline Primary school & $33(30.6 \%)$ & $36(35.6 \%)$ & \\
\hline Junior high school & $33(30.6 \%)$ & $33(32.7 \%)$ & \\
\hline High school/technical secondary school & $20(18.5 \%)$ & $20(19.8 \%)$ & \\
\hline College degree and above & $12(11.2 \%)$ & $\mathrm{I}(\mathrm{I} .0 \%)$ & \\
\hline Insurance, n (\%) & & & 0.779 \\
\hline Local medical insurance & $53(48.9 \%)$ & $55(54.5 \%)$ & \\
\hline Offsite medical insurance & $36(33.3 \%)$ & $27(26.7 \%)$ & \\
\hline No medical insurance & $19(17.6 \%)$ & $19(18.8 \%)$ & \\
\hline \multicolumn{4}{|l|}{ Risk factors, n (\%) } \\
\hline Smoking & $49(45.4 \%)$ & $44(43.6 \%)$ & 0.793 \\
\hline Family history of cardiovascular and cerebrovascular diseases & $6(5.6 \%)$ & $8(7.9 \%)$ & 0.494 \\
\hline TIA & I (0.9\%) & $2(2.0 \%)$ & $0.953^{\dagger}$ \\
\hline Hypertension & $73(67.6 \%)$ & $70(69.3 \%)$ & 0.790 \\
\hline Diabetes mellitus & $28(25.9 \%)$ & $33(32.7 \%)$ & 0.284 \\
\hline Atrial fibrillation & $3(2.8 \%)$ & $9(8.9 \%)$ & $0.057^{\dagger}$ \\
\hline Coronary heart disease & $5(4.6 \%)$ & $4(4.0 \%)$ & $0.8 \mathrm{II}^{\dagger}$ \\
\hline Hyperhomocysteinemia & $23(21.3 \%)$ & 21 (20.8\%) & 0.929 \\
\hline Hyperlipidemia & $19(17.6 \%)$ & $19(18.8 \%)$ & 0.819 \\
\hline MAS, median (IQR) & $12.00(4.00-24.80)$ & II.00 (3.00-24.50) & 0.604 \\
\hline FMA, median (IQR) & $28.50(17.00-53.80)$ & $30.00(12.00-53.50)$ & 0.603 \\
\hline ARAT, median (IQR) & $1.00(0.00-26.80)$ & $0.00(0.00-28.50)$ & 0.673 \\
\hline MBI, median (IQR) & $29.50(20.70-50.00)$ & $28.00(20.00-55.00)$ & $0.929^{\ddagger}$ \\
\hline NIHSS, median (IQR) & $6.00(4.00-9.00)$ & $6.00(3.00-10.00)$ & $0.613^{\ddagger}$ \\
\hline mRS, median (IQR) & $4.00(4.00-4.00)$ & $4.00(4.00-4.00)$ & 0.992 \\
\hline
\end{tabular}

Notes: ${ }^{\dagger}$ Fisher exact probability method; ${ }^{\ddagger}$ Mann-Whitney U-test.

Abbreviations: wCST-LL, weighted corticospinal tract lesion load; TIA, transient ischemic attacks; MAS, Motor Assessment Scale; FMA, FugI-Meyer Assessment; ARAT, Action Research Arm Test; MBI, modified Barthel Index; NIHSS, National Institutes of Health Stroke Scale; mRS, modified Rankin Scale; SD, standard deviation; IQR, interquartile range.

The post hoc analysis involved Friedman test in determining the statistically significant simple effect for factor time in MBI scores within the groups $(P<0.001))$ and the statistically significant main effect for factor time in NIHSS $(P<0.001)$ and mRS $(P<0.001)$ scores of the study subjects. The Bonferroni corrected pairwise comparison detected statistically significant differences except for in mRS between t0 and t1 ( $P=0.141$ after adjustment) (Supplementary Table S3). 

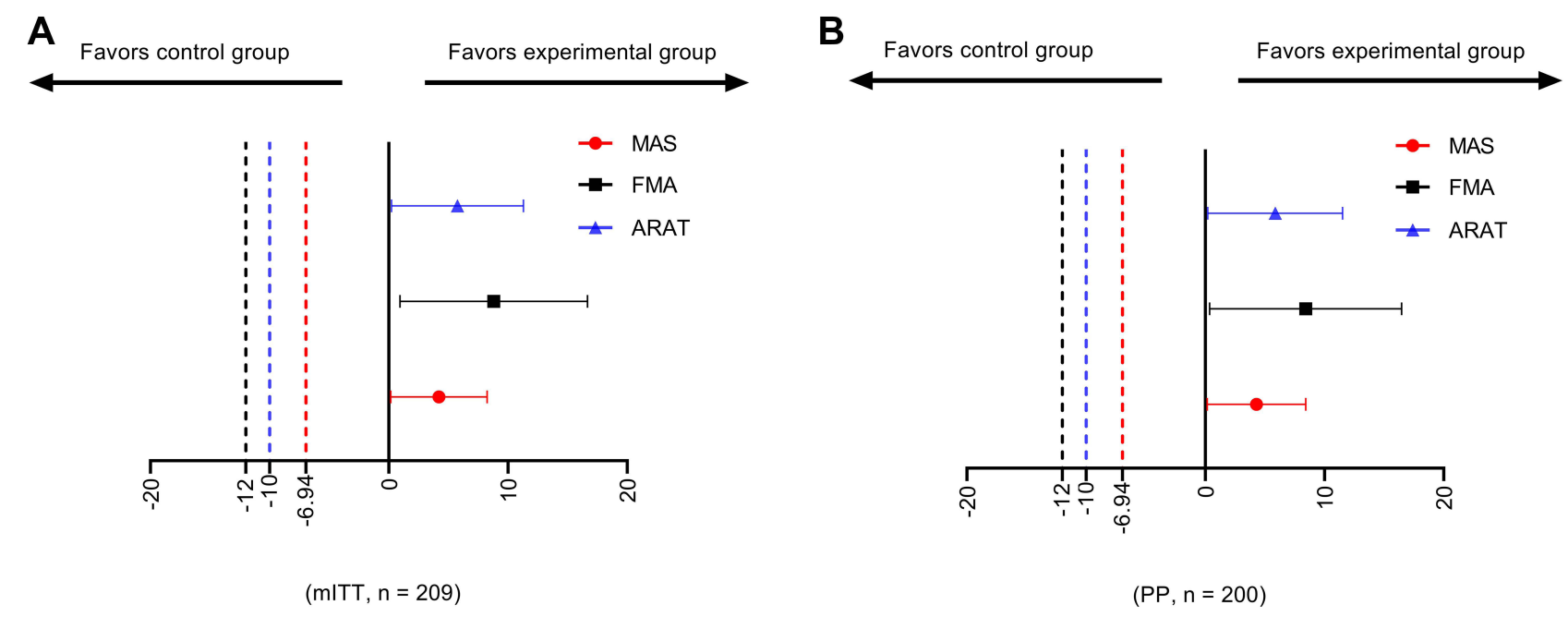

Figure 2 Treatment difference and non-inferiority margin. (A) Non-inferiority test for the modified intention-to-treat dataset, $\mathrm{n}=209$. (B) Non-inferiority test for the perprotocol dataset, $\mathrm{n}=200$.

Notes: Data are means (symbols) and $95 \% \mathrm{Cls}$ (error bars) for the primary outcome difference (T-C) between nurse-led (the test group, $\mathrm{T}$ ) and therapist-led (the control group, C) rehabilitation after seven days of treatment. The dotted lines represent the non-inferiority margins $(\delta)$.

Abbreviations: MAS, Motor Assessment Scale, $\delta=-6.94$; FMA, Fugl-Meyer Assessment, $\delta=-12$; ARAT, Action Research Arm Test, $\delta=-10$.

\section{Adverse Events}

During the study period, one patient died at 12 weeks' follow-up, and none of the patients had other severe adverse events. Four patients dropped out because the disease worsened in the experimental group. The exacerbations were not related to the interventions, and these participants were excluded from the effectiveness analysis.

\section{Discussion}

This study was a seven-day rehabilitation nursing intervention of motor function for AIS, it appeared to be non- inferior and not superior to therapist-led usual rehabilitation. The ADL, severity of stroke and disability significantly changed over time but were similar between the groups. The ADL changes may be more significant in the experimental group over time. Because this was an exploratory trial, no definitive conclusion could be drawn regarding the effectiveness of rehabilitation nursing; nevertheless, it appeared to be a safe care pathway for AIS rehabilitation.

This was the first study in patients with AIS to use a non-inferiority trial design to evaluate the efficacy of

Table 2 Between-Group Difference on Primary Outcomes After 7 Days Treatment (PP Analysis, n=200)

\begin{tabular}{|c|c|c|c|c|c|c|c|}
\hline \multirow[t]{2}{*}{ Outcome Measure } & \multirow[t]{2}{*}{$\begin{array}{l}\text { Experimental Group } \\
(\mathrm{n}=105)\end{array}$} & \multirow[t]{2}{*}{$\begin{array}{l}\text { Control Group } \\
(n=95)\end{array}$} & \multicolumn{3}{|c|}{ Noninferiority I-Tailed Test ${ }^{a}$} & \multicolumn{2}{|c|}{$\begin{array}{l}\text { Superiority } \\
\text { 2-Tailed Test }\end{array}$} \\
\hline & & & Non-Inferiority Margin ( $\delta$ ) & $\mathbf{Z}$ & $P$ value & $\mathbf{Z}$ & $P$ value \\
\hline MAS & $24.00(9.00-38.00)$ & $13.00(5.00-32.00)$ & 6.94 & -5.222 & $<0.00 I^{*}$ & -2.174 & 0.030 \\
\hline FMA & $51.00(20.50-79.00)$ & $33.00(17.00-68.00)$ & 12.00 & -4.929 & $<0.00 I^{*}$ & -1.982 & 0.047 \\
\hline ARAT & $17.00(0.00-39.00)$ & $0.00(0.00-34.00)$ & 10.00 & -6.245 & $<0.00 I^{*}$ & -1.913 & 0.056 \\
\hline Change in MAS & $5.00(1.50-14.00)$ & $3.00(0.00-7.00)$ & 2.15 & -5.815 & $<0.00 I^{*}$ & -3.012 & $0.003 *$ \\
\hline Change in FMA & $7.50(1.25-24.50)$ & $5.00(0.00-9.00)$ & 3.62 & -5.577 & $<0.00 I^{*}$ & -2.878 & $0.004 *$ \\
\hline Change in ARAT & $1.00(0.00-17.00)$ & $0.00(0.00-4.00)$ & 3.65 & -7.841 & $<0.00 I^{*}$ & -3.265 & $0.00 I^{*}$ \\
\hline
\end{tabular}

Notes: Outcome measures presented as median (IQR); overall alpha is 0.025 for both noninferiority and superiority testing; *test on outcome difference within the given noninferiority margins or have superiority $(P \leq 0.05 / 3=0.0167)$; ${ }^{a}$ Mann-Whitney $U$ or Wilcoxon Rank-Sum Location Difference Test for Non-Inferiority; ${ }^{b} M a n n-W h i t n e y$ U-Test for Two Independent Samples.

Abbreviations: IQR, interquartile range; MAS, Motor Assessment Scale; FMA, Fugl-Meyer Assessment; ARAT, Action Research Arm Test. 


\begin{tabular}{|c|c|c|c|c|}
\hline 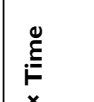 & 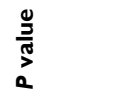 & $\frac{*}{8}$ & $\begin{array}{l}\bar{\infty} \\
\text { O্. }\end{array}$ & $\frac{\text { in }}{0}$ \\
\hline 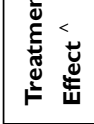 & 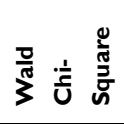 & $\underset{\substack{a \\
\underline{0}}}{\underline{0}}$ & $\underset{\substack{n \\
6}}{\stackrel{n}{6}}$ & $\frac{2}{n}$ \\
\hline \multirow{2}{*}{ 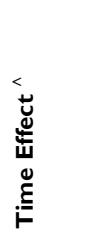 } & $\frac{\mathscr{g}}{\frac{2}{N}}$ & $\begin{array}{l}\frac{*}{8} \\
\dot{0} \\
\mathrm{v}\end{array}$ & $\begin{array}{l}\frac{*}{8} \\
\dot{0} \\
\mathrm{v}\end{array}$ & $\begin{array}{l}\frac{*}{8} \\
\dot{0} \\
\dot{v}\end{array}$ \\
\hline & 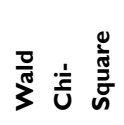 & $\begin{array}{l}\bar{y} \\
\dot{q} \\
\dot{\xi}\end{array}$ & $\begin{array}{l}\hat{\infty} \\
\stackrel{\infty}{0} \\
\hat{\circ}\end{array}$ & $\begin{array}{c}\stackrel{m}{o} \\
\underset{N}{N}\end{array}$ \\
\hline \multirow{2}{*}{ 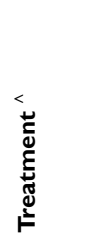 } & 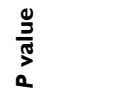 & 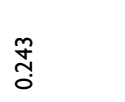 & $\frac{0}{\mathfrak{n}}$ & ڤ్ \\
\hline & 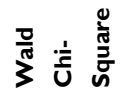 & ָֻ & ্ָণ্ণ & $\frac{0}{i}$ \\
\hline \multirow[b]{2}{*}{ 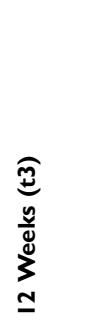 } & 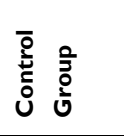 & 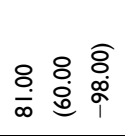 & $\begin{array}{l}8 \\
\stackrel{0}{0} \\
\stackrel{8}{8} \\
\stackrel{i}{-}\end{array}$ & $\begin{array}{l}\stackrel{8}{d} \\
\stackrel{i}{8} \\
\stackrel{8}{\dot{m}}\end{array}$ \\
\hline & 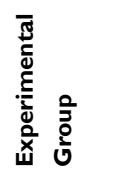 & 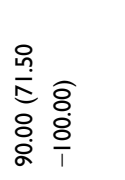 & $\frac{8}{\dot{0}}$ & 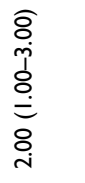 \\
\hline \multirow[b]{2}{*}{ 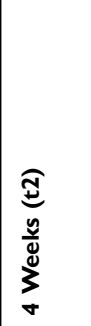 } & $\begin{array}{ll}\overline{0} & 0 \\
\dot{t} & 0 \\
0 & 0 \\
0 & 0\end{array}$ & 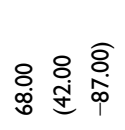 & 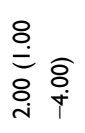 & 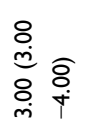 \\
\hline & 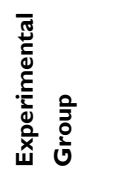 & 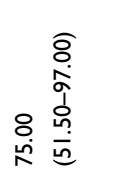 & $\begin{array}{l}\widetilde{8} \\
\dot{1} \\
0 \\
0 \\
0 \\
\stackrel{i}{i}\end{array}$ & 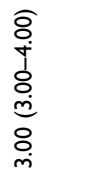 \\
\hline \multirow[b]{2}{*}{ 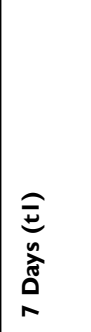 } & $\begin{array}{ll}\overline{0} & 0 \\
& 0 \\
0 & 0 \\
0 & 0\end{array}$ & 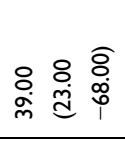 & 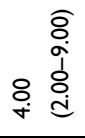 & 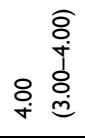 \\
\hline & 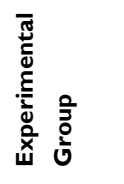 & 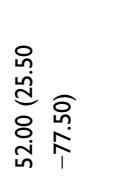 & 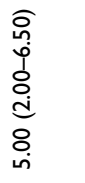 & $\begin{array}{l}\text { ọ } \\
\dot{1} \\
0 \\
\dot{m} \\
8 \\
\dot{+}\end{array}$ \\
\hline \multirow[b]{2}{*}{ 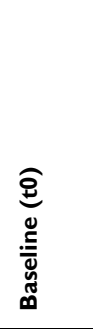 } & $\begin{array}{ll}\overline{0} & 0 \\
\grave{2} & 0 \\
0 & 0 \\
0 & 0\end{array}$ & 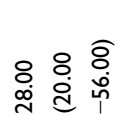 & $\begin{array}{l}8 \\
\stackrel{8}{0} \\
\stackrel{0}{8} \\
\stackrel{0}{0}\end{array}$ & 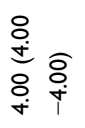 \\
\hline & 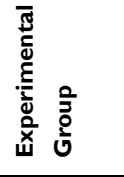 & 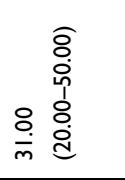 & 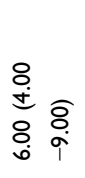 & $\begin{array}{l}\stackrel{8}{ \pm} \\
\stackrel{+}{8} \\
\dot{+}\end{array}$ \\
\hline 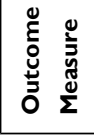 & & $\overline{\underline{\alpha}}$ & $\begin{array}{l}\text { 品 } \\
\bar{\Sigma}\end{array}$ & $\stackrel{\widetilde{\alpha}}{\varepsilon}$ \\
\hline
\end{tabular}

rehabilitation nursing versus a proven effective therapistled treatment. Non-inferiority for intervention effects was demonstrated to improve motor function recovery in AIS in this study. It is unclear about the MCID of the MAS scores of stroke survivors in early rehabilitation studies. The MCID for the FMA was 6.6 and 6 points for the upper and lower extremity respectively. ${ }^{40}$ We assumed that the $\delta$ of the FMA was 12, which was below the MCID. Research has shown that the MCID values for the ARAT were 12 and 17 points for upper extremity standardized measures early post-stroke. ${ }^{41}$ Assuming a delta of 6.94 in the MAS and 10 in the ARAT, the non-inferiority test corresponded to the power of $0.896,0.925$, and 0.817 for the MAS, ARAT and FMA respectively in this study and should be considered to be stringent.

The improvement in motor function favored the nurseled rehabilitation although there was no statistical difference. The results of interaction group and time effect suggest that short-term rehabilitation treatment in the acute phase has only some long-term effects on ADLs. Consistent findings came from the fact that the difference in the effect of early mobilization on mRS scores $0-2$ was not statistically significant but did improve the Barthel Index. ${ }^{42}$ These results can be explained by the difference between rehabilitation the nursing program and therapistled rehabilitation. ${ }^{33}$ During the study period, the intensity and content of rehabilitation treatment were not uniform. In terms of training content, the discrepancy between function outcomes is unsurprising considering that patients were graded according to their MBI scores and were provided with task-oriented and individualized rehabilitation intervention programs at the corresponding level. The task-oriented rehabilitation nursing program was in line with stroke guidelines and principles of care. ${ }^{43}$ The program resembled a research that includes individualized patient schedules, independent practice, and increased patient activity during inpatient stroke rehabilitation. ${ }^{44}$ The present study took advantage of a high-compliance design, and the checklist format of the training items in the program facilitated the implementation and improved the intensity of rehabilitation. Whereas another acute phase trial (AMOBES) found that the dose of physiotherapy had similar benefits for limb impairment, our intervention differed in that not only PT was involved. ${ }^{45}$ Moreover, motor function assessments are very sensitive and specific to determining the level of impairment and recovery. The FMA has a ceiling effect, while the ARAT has a floor 
effect. ${ }^{46}$ Combination with MAS strengthens the observed effects.

The nurse-led rehabilitation intervention program was effective in improving motor function and ADL without significant adverse events. The intervention components of our program were appropriate, it was consistent with an inpatient settings rehabilitation guideline implementation study, which evaluated seven treatments implemented by nurses, including the range of motion and/or stretching, task-oriented training, and sit-to-stand training. ${ }^{47}$ This study responds to the advocacy for advanced nursing practice care pathways in the AIS, and explores the relationship between nursing interventions and clinical outcomes. $^{48}$ As previously described, not all patients can obtain rehabilitation services in the hospital, even among the patients receiving rehabilitation. The most common therapy is isolated PT for motor dysfunction. ${ }^{2}$ The current strength training program relied on clinical judgment rather than following guiding principles. ${ }^{49}$ Thus, there are no standard physiotherapeutic measures; therapists determine training programs appropriate to the case or condition based on susceptibility and consensus with patients and caregivers.

Nurses encourage patients to perform meaningful tasks, achieve favorable functional outcomes, ${ }^{24}$ and play a central role in stroke recovery in the acute setting. ${ }^{21,29}$ Nurses fulfilled the role of a professional practitioner, case manager, and researcher of rehabilitation as required by the association of rehabilitation nursing. ${ }^{50}$ It also improved professional competence for nurses and may demonstrate that they can complement multidisciplinary teams in areas or periods where rehabilitation resources are insufficient.

It is important to emphasize that there is no role conflict between nurses and physiotherapists in stroke rehabilitation and that exercise training as part of the care is necessary. Because in many parts of the world, stroke patients do not have access to formal rehabilitation under their current health care systems, nurses may be the only hope for these patients. ${ }^{51}$ Nurse-led services may be more effective in improving patient adherence to evidence-based recommendations. ${ }^{52}$ Nurse can integrate the rehabilitation activities into the patient's daily routine, and may not add their workload. ${ }^{21}$ Nevertheless, nurse-led programs are uncommon in stroke care. Rehabilitation nursing practice may be hindered by misunderstood roles and underappreciation from therapists or other professionals, the need for long-term knowledge and skills training, ${ }^{22}$ and the need to balance the physical care needs with rehabilitation needs of stroke survivors. ${ }^{26}$

This study has some limitations. First, placebocontrolled trials should be performed to demonstrate the intervention's effectiveness, which has been addressed in a pilot study and considered for reporting elsewhere. However, for ethical reasons, a three-arm trial including a placebo control group could not be achieved in the present study. Second, the intervention duration was seven days, a relatively short time limited by the average LOS of AIS patients in China. Though the average LOS was longer than the US median LOS of 4 days. ${ }^{53}$ Followup lasted 12 weeks, which was sufficient because the improvement in motor function is more significant in the first four months, and there may not be much progress after that. ${ }^{54}$ Finally, this was a single-center trial. Therefore, the generalization of findings to other populations has yet to be validated. In the future, more robust research should be encouraged to extend the findings of this study. Cost-effectiveness analysis and self-care assessment should be followed.

\section{Conclusion}

The intervention of the rehabilitation nursing program graded according to the MBI appeared to be non-inferior to therapist-led treatment. Preliminary analysis of the repeated-measures data showed that differences in prognosis and neurological deficits between the two groups were not statistically significant, with only interaction of intervention and time on the MBI. Both rehabilitation treatments appeared to be safe and effective. However, no definitive conclusions could be drawn as to the nature of the exploratory research. It is necessary to conduct independently replicated studies with large samples to validate our findings. Identifying and developing effective rehabilitation nursing interventions is essential for aging populations, especially those in LMICs.

\section{Data Sharing Statement}

The authors do not intend to share individual de-identified participant data.

\section{Acknowledgments}

We appreciate the multidisciplinary team from the hospital, especially the nurses who contributed to the implementation of this study, and the statistician Zexin Chen for the support. We also want to thank all patients participated in this study 
and the rehabilitation experts who were dedicated to developing the intervention program.

\section{Funding}

This study was supported by the National Natural Science Foundation of China (no: 81871839).

\section{Disclosure}

The authors report no conflicts of interest in this work.

\section{References}

1. Krishnamurthi RV, Ikeda T, Feigin VL. Global, regional and country-specific burden of ischaemic stroke, intracerebral haemorrhage and subarachnoid haemorrhage: a systematic analysis of the global burden of disease study 2017. Neuroepidemiology. 2020;54 (2):171-179. doi: $10.1159 / 000506396$

2. Wu S, Wu B, Liu M, et al. Stroke in China: advances and challenges in epidemiology, prevention, and management. Lancet Neurol. 2019;18(4):394-405. doi:10.1016/S1474-4422(18)30500-3

3. Scrutinio D, Giardini A, Chiovato L, et al. The new frontiers of rehabilitation medicine in people with chronic disabling illnesses. Eur J Intern Med. 2019;61:1-8. doi:10.1016/j.ejim.2018.10.019

4. United Nations DoE, Social Affairs PD. World population ageing 2019: highlights (ST/ESA/SER. A/430). 2019.

5. Ntaios G, Michel P, Georgiopoulos G, et al. Characteristics and outcomes in patients with COVID-19 and acute ischemic stroke: the global COVID-19 stroke registry. Stroke. 2020;51(9):e254 e258. doi:10.1161/STROKEAHA.120.031208

6. Wang CC, Chao JK, Wang ML, et al. Care for patients with stroke during the COVID-19 pandemic: physical therapy and rehabilitation suggestions for preventing secondary stroke. J Stroke Cerebrovasc Dis. 2020;29:105182. doi:10.1016/j.jstrokecerebrovasdis.2020.105182

7. Legg LA, Lewis SR, Schofield-Robinson OJ, Drummond A, Langhorne P. Occupational therapy for adults with problems in activities of daily living after stroke. Cochrane Database Syst Rev. 2017;7: CD003585.

8. Cumming TB, Churilov L, Collier J, et al. Early mobilization and quality of life after stroke: findings from AVERT. Neurology. 2019;93 (7):e717-e728. doi:10.1212/WNL.0000000000007937

9. Pramanick J, Uchat U, Chattopadhyay A, Mir AA, Koley M, Saha S. An open-label randomized pragmatic non-inferiority pilot trial comparing the effectiveness of Curare $30 \mathrm{CH}$ against individualized homeopathic medicines in post-stroke hemiparesis. Adv Integr Med. 2020;7(2):79-88. doi:10.1016/j.aimed.2019.06.002

10. Powers WJ, Rabinstein AA, Ackerson T, et al. Guidelines for the early management of patients with acute ischemic stroke: 2019 update to the 2018 guidelines for the early management of acute ischemic stroke: a guideline for healthcare professionals from the American Heart Association/American Stroke Association. Stroke. 2019;50(12):e344-e418. doi:10.1161/STR.0000000000000211

11. Fan YT, Lin KC, Liu HL, Chen YL, Wu CY. Changes in structural integrity are correlated with motor and functional recovery after post-stroke rehabilitation. Restor Neurol Neurosci. 2015;33 (6):835-844. doi:10.3233/RNN-150523

12. Teasell R, Salbach NM, Foley N, et al. Canadian stroke best practice recommendations: rehabilitation, recovery, and community participation following stroke. 6th edition update 2019. Int J Stroke. 2020;15:763-788. doi:10.1177/1747493019897843

13. Livingston-Thomas J, Nelson P, Karthikeyan S, et al. Exercise and environmental enrichment as enablers of task-specific neuroplasticity and stroke recovery. Neurotherapeutics. 2016;13(2):395-402. doi:10.1007/s13311-016-0423-9
14. Bernhardt J, Godecke E, Johnson L, Langhorne P. Early rehabilitation after stroke. Curr Opin Neurol. 2017;30(1):48-54. doi:10.1097/ WCO.0000000000000404

15. Spoon D, Rietbergen T, Huis A, et al. Implementation strategies used to implement nursing guidelines in daily practice: a systematic review. Int $J$ Nurs Stud. 2020;111:103748. doi:10.1016/j. ijnurstu.2020.103748

16. Eng JJ, Bird ML, Godecke E, et al. Moving stroke rehabilitation research evidence into clinical practice: consensus-based core recommendations from the stroke recovery and rehabilitation roundtable. Neurorehab Neural Repair. 2019;33(11):935-942. doi:10.1177/ 1545968319886485

17. Bernhardt J, Urimubenshi G, Gandhi DBC, Eng JJ. Stroke rehabilitation in low-income and middle-income countries: a call to action. Lancet. 2020;396(10260):1452-1462. doi:10.1016/S0140-6736(20)31313-1

18. Xie L, Xu T, Liu Y, et al. A study on the current status of domestic rehabilitation therapy education (in Chinese). China Rehabilitation. 2019;10. doi:CNKI:SUN:ZLKF.0.2019-10-023

19. Gimigliano F, Negrini S. The World Health Organization "Rehabilitation 2030: a call for action". Eur J Phys Rehabil Med. 2017;53(2):155-168. doi:10.23736/S1973-9087.17.04746-3

20. Dee M, Lennon O, O'Sullivan C. A systematic review of physical rehabilitation interventions for stroke in low and lower-middle income countries. Disabil Rehabil. 2020;42(4):473-501. doi:10.1080/09638288.2018.1501617

21. Woon C. Nursing at the centre of stroke recovery in the acute setting: prioritising early rehabilitation. $B r J$ Neurosci Nurs. 2016;12 (1):23-28. doi:10.12968/bjnn.2016.12.1.23

22. Gutenbrunner C, Stievano A, Nugraha B, Stewart D, Catton H. Nursing - a core element of rehabilitation. Int Nurs Rev. 2021. doi:10.1111/inr.12661

23. Harris T, Kerry SM, Victor CR, et al. A primary care nurse-delivered walking intervention in older adults: PACE (pedometer accelerometer consultation evaluation)-Lift cluster randomised controlled trial. PLoS Med. 2015;12(2):e1001783. doi:10.1371/journal.pmed.1001783

24. Tijsen LM, Derksen EW, Achterberg WP, Buijck BI. Challenging rehabilitation environment for older patients. Clin Interv Aging. 2019;14:1451-1460. doi:10.2147/CIA.S207863

25. Richards DA, Hilli A, Pentecost C, Goodwin VA, Frost J. Fundamental nursing care: a systematic review of the evidence on the effect of nursing care interventions for nutrition, elimination, mobility and hygiene. J Clin Nurs. 2018;27(11-12):2179-2188. doi:10.1111/jocn.14150

26. Clarke DJ. Nursing practice in stroke rehabilitation: systematic review and meta-ethnography. J Clin Nurs. 2014;23(9-10):1201-1226. doi:10.1111/jocn.12334

27. Bettger JP, Li ZX, Xian Y, et al. Assessment and provision of rehabilitation among patients hospitalized with acute ischemic stroke in China: findings from the China National Stroke Registry II. Int J Stroke. 2017;12(3):254-263. doi:10.1177/1747493017701945

28. Wang J, Chen Y, Zhang Y, Li M, Jin J. Rehabilitation nursing for motor functional recovery of acute ischaemic stroke: study protocol for a randomised controlled trial. BMJ Open. 2020;10(9):e037391. doi:10.1136/bmjopen-2020-037391

29. Abd El-Hay SA, Abed Allah AK, Tag El Din EA. Effect of implementing designed educational training program for neurological nurses on clinical outcomes of stroke patients. Clini Nurs Stud. 2018;6(4):121. doi:10.5430/cns.v6n4p121

30. Liu H, Zhu D, Song B, et al. Cost-effectiveness of an intervention to improve the quality of nursing care among immobile patients with stroke in China: a multicenter study. Int $J$ Nurs Stud. 2020;110:103703. doi:10.1016/j.ijnurstu.2020.103703

31. Piaggio G, Elbourne DR, Pocock SJ, Evans SJW, Altman DG. Reporting of noninferiority and equivalence randomized trials: extension of the CONSORT 2010 statement. JAMA. 2012;308 (24):2594-2604. doi:10.1001/jama.2012.87802 
32. Angeli F, Verdecchia P, Vaudo G, Masnaghetti S, Reboldi G. Optimal use of the non-inferiority trial design. Pharmaceut Med. 2020;34 (3):159-165. doi:10.1007/s40290-020-00334-z

33. Jin J, Li M, Chen Y, et al. Construction of early motor rehabilitation nursing program based on Modified Barthel Index grading for stroke patients (in Chinese). Chin J Nurs. 2020;55(09):1360-1365. doi:10.3761/j.issn.0254-1769.2020.09.016

34. Liu H, Zhu C, Cao J, et al. Hospitalization costs among immobile patients with hemorrhagic or ischemic stroke in China: a multicenter cross-sectional study. BMC Health Serv Res. 2020;20(1):905. doi:10.1186/s12913-020-05758-6

35. Chippala P, Sharma R. Effect of very early mobilisation on functional status in patients with acute stroke: a single-blind, randomized controlled trail. Clin Rehabil. 2015;30(7):669-675. doi:10.1177/ 0269215515596054

36. Carr JH, Shepherd RB, Nordholm L, Lynne D. Investigation of a new motor-assessment scale for stroke patients. Phys Ther. 1985;65 (2):175-180. doi:10.1093/ptj/65.2.175

37. Gladstone DJ, Danells CJ, Black SE. The Fugl-Meyer Assessment of motor recovery after stroke: a critical review of its measurement properties. Neurorehabil Neural Repair. 2002;16(3):232-240. doi:10.1177/154596802401105171

38. Nijland $\mathrm{R}$, van Wegen E, Verbunt J, van Wijk R, van Kordelaar J, Kwakkel G. A comparison of two validated tests for upper limb function after stroke: the Wolf Motor Function Test and the Action Research Arm Test. J Rehabil Med. 2010;42(7):694-696. doi:10.2340/16501977-0560

39. Horton EJ, Mitchell KE, Johnson-Warrington V, et al. Comparison of a structured home-based rehabilitation programme with conventional supervised pulmonary rehabilitation: a randomised non-inferiority trial. Thorax. 2018;73(1):29-36. doi:10.1136/thoraxjnl-2016-208506

40. Lim JY, Oh MK, Park J, Paik NJ. Does measurement of corticospinal tract involvement add value to clinical behavioral biomarkers in predicting motor recovery after stroke? Neural Plast. 2020;2020:8883839. doi:10.1155/2020/8883839

41. Lang CE, Edwards DF, Birkenmeier RL, Dromerick AW. Estimating minimal clinically important differences of upper-extremity measures early after stroke. Arch Phys Med Rehabil. 2008;89(9):1693-1700. doi:10.1016/j.apmr.2008.02.022

42. Li Z, Zhang X, Wang K, Wen J. Effects of early mobilization after acute stroke: a meta-analysis of randomized control trials. $J$ Stroke Cerebrovasc Dis. 2018;27(5):1326-1337. doi:10.1016/j. jstrokecerebrovasdis.2017.12.021

43. Wolf SL, Winstein CJ, Miller JP, et al. Effect of constraint-induced movement therapy on upper extremity function 3 to 9 months after stroke - the EXCITE randomized clinical trial. JAMA. 2006;296 (17):2095-2104. doi:10.1001/jama.296.17.2095
44. Tyson SF, Burton L, McGovern A. The effect of a structured programme to increase patient activity during inpatient stroke rehabilitation: a phase I cohort study. Clin Rehabil. 2016;30(2):191-198. doi: $10.1177 / 0269215515575335$

45. Yelnik AP, Quintaine V, Andriantsifanetra C, et al. AMOBES (Active Mobility Very Early After Stroke): a randomized controlled trial. Stroke. 2017;48(2):400-405. doi:10.1161/STROKEAHA.116.014803

46. Findlater SE, Hawe RL, Mazerolle EL, et al. Comparing CST lesion metrics as biomarkers for recovery of motor and proprioceptive impairments after stroke. Neurorehab Neural Repair. 2019;33 (10):848-861. doi:10.1177/1545968319868714

47. Salbach NM, Wood-Dauphinee S, Desrosiers J, et al. Facilitated interprofessional implementation of a physical rehabilitation guideline for stroke in inpatient settings: process evaluation of a cluster randomized trial. Implement Sci. 2017;12(1):100. doi:10.1186/ s13012-017-0631-7

48. Laird EA, McCauley C, Ryan A, Beattie A. 'The lynchpin of the acute stroke service'-an envisioning of the scope and role of the advanced nurse practitioner in stroke care in a qualitative study. J Clin Nurs. 2020;29(23-24):4795-4805. doi:10.1111/jocn.15523

49. Tole G, Raymond MJ, Williams G, Clark RA, Holland AE. Strength training to improve walking after stroke: how physiotherapist, patient and workplace factors influence exercise prescription. Physiother Theory Pract. 2020;1-9. doi:10.1080/09593985.2020.1839986

50. Vaughn S, Mauk KL, Jacelon CS, et al. The competency model for professional rehabilitation nursing. Rehabil Nurs. 2016;41(1):33-44. doi:10.1002/rnj.225

51. Tseng CN, Chen CCH, Wu SC, Lin LC. Effects of a range-of-motion exercise programme. J Adv Nurs. 2007;57(2):181-191. doi:10.1111/ j.1365-2648.2006.04078.x

52. O'Toole K, Chamberlain D, Giles T. Exploration of a nurse practitioner-led phase two cardiac rehabilitation programme on attendance and compliance. $J$ Clin Nurs. 2020;29(5-6):785-793. doi:10.1111/jocn. 15133

53. Winstein CJ, Stein J, Arena R, et al. Guidelines for adult stroke rehabilitation and recovery a guideline for healthcare professionals from the American Heart Association/American Stroke Association. Stroke. 2016;47(6):e98-e169. doi:10.1161/STR.0000000000000098

54. Winstein CJ, Wolf SL, Dromerick AW, et al. Effect of a task-oriented rehabilitation program on upper extremity recovery following motor stroke: the ICARE randomized clinical trial. JAMA. 2016;315 (6):571-581. doi:10.1001/jama.2016.0276
Clinical Interventions in Aging

\section{Publish your work in this journal}

Clinical Interventions in Aging is an international, peer-reviewed journal focusing on evidence-based reports on the value or lack thereof of treatments intended to prevent or delay the onset of maladaptive correlates of aging in human beings. This journal is indexed on PubMed Central, MedLine, CAS, Scopus and the Elsevier
Bibliographic databases. The manuscript management system is completely online and includes a very quick and fair peer-review system, which is all easy to use. Visit http://www.dovepress.com/ testimonials.php to read real quotes from published authors. 